\title{
Horizontal and Vertical Rule Bases Method in Fuzzy Controllers
}

\author{
Sadegh Aminifar and Arjuna bin Marzuki \\ School of Electrical and Electronics Engineering, Universiti Sains Malaysia (USM), Engineering Campus, 14300 Nibong Tebal, \\ Pulau Pinang, Malaysia \\ Correspondence should be addressed to Sadegh Aminifar; sadeghaminifar@yahoo.com
}

Received 12 January 2013; Revised 8 February 2013; Accepted 12 February 2013

Academic Editor: Xiaojie Su

Copyright (C) 2013 S. Aminifar and A. bin Marzuki. This is an open access article distributed under the Creative Commons Attribution License, which permits unrestricted use, distribution, and reproduction in any medium, provided the original work is properly cited.

\begin{abstract}
Concept of horizontal and vertical rule bases is introduced. Using this method enables the designers to look for main behaviors of system and describes them with greater approximations. The rules which describe the system in first stage are called horizontal rule base. In the second stage, the designer modulates the obtained surface by describing needed changes on first surface for handling real behaviors of system. The rules used in the second stage are called vertical rule base. Horizontal and vertical rule bases method has a great roll in easing of extracting the optimum control surface by using too lesser rules than traditional fuzzy systems. This research involves with control of a system with high nonlinearity and in difficulty to model it with classical methods. As a case study for testing proposed method in real condition, the designed controller is applied to steaming room with uncertain data and variable parameters. A comparison between PID and traditional fuzzy counterpart and our proposed system shows that our proposed system outperforms PID and traditional fuzzy systems in point of view of number of valve switching and better surface following. The evaluations have done both with model simulation and DSP implementation.
\end{abstract}

\section{Introduction}

In this research we want to propose a control system which has good flexibility with uncertain data by introducing vertical and horizontal concept in order to control the temperature of autoclave steam room of industrial stone manufactures which is a system with high nonlinearity and unforeseeable parameters [1].

There are several different methods for handling uncertainty applicable in stochastic conditions [2-7], but none of them led to simpler implementation than traditional fuzzy systems. Innovations in new methods for control with malfunctioned elements in real industrial environments are the aim of this paper in order to increase the quality of product and increase energy efficiency. This paper wants to supervise the complicated system via expert human knowledge which describes the system with words and controls it according to the rules which are extracted from knowledge base.

For the second stage, the nonlinearity of system is found with identification of desired curve. Identification of real systems is a fundamental task in science and engineering applications because we can obtain models of systems with real input/output data. In control applications it is very common to use models of dynamic systems in the sense of models of systems with memory; they can use past, present, and future samples' values of sequences. Hence, we decided to include our proposed nonlinear prediction problem [8-10].

Fuzzy type II systems are the existent model for handling these uncertainties [11-13]. However, there are still various deficiencies regarding fuzzy type II systems to model the crisp data as words and handling the uncertainties especially involving a system with high nonlinearities and real probabilistic space expectation in the output. The new method which is proposed in this paper to implement controller of the autoclave system has almost all advantages of the other three methods that are on-off, PID, and conventional fuzzy method. It controls the rate of valve opening in the condition where unforeseen variations are high. Valve amortization is minimized; meanwhile, it is simple and inexpensive $[14,15]$.

After designing the total system, the system is simulated with theoretical data and finally it is verified by applying to real steaming room. 


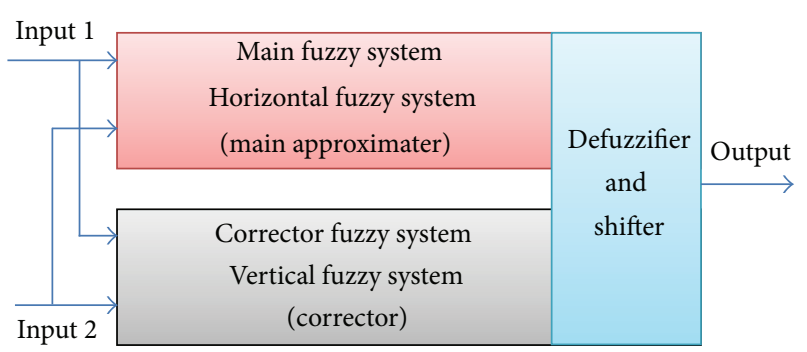

FIGURE 1: Vertical and horizontal fuzzy rule bases structure.

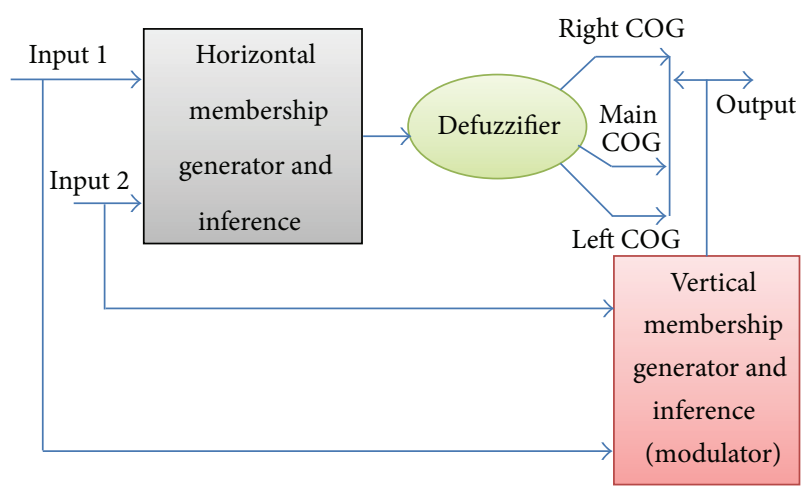

FIGURE 2: The concept of horizontal and vertical rule bases.

\section{Horizontal and Vertical Rule Base Concept}

An $n+1$ dimension surface describes the relation between inputs and output of every fuzzy system with several inputs and one output. The great concern is to implement the mentioned surface more proper with the behavior of plant under process. This paper contributes to implement this surface with lesser rules especially in the time in which process of plant behavior is complicated and system data are more uncertain. In this method the system is described in two stages. First, the designer looks for main behaviors of system and describes them with greater approximations. The rules which describe the system in the first stage are called horizontal rule bases. In the second stage, the designer modulates the obtained surface from first stage by describing needed changes on first surface for handling real behaviors of system. The rules used in second stage are called vertical rule bases. Figure 1 shows that finding main route and rectifying it are done simultaneously.

Figure 2 shows that the vertical rule base shifts the point of main center of gravity of output fuzzy word. The possible range for obtaining output via proposed system is between left center of gravity and right center of gravity which was shown in Figure 3. Left COG is the center of gravity of left side of main COG vertical line and right COG is located on the right of this line as shown in Figure 3. The "Band Width" is the distance between left and right COGs as shown in Figure 3. Our used strategy for vertical band width was shown in Figure 3 which is not absolute definition for possible range of output to be used by vertical rule base.

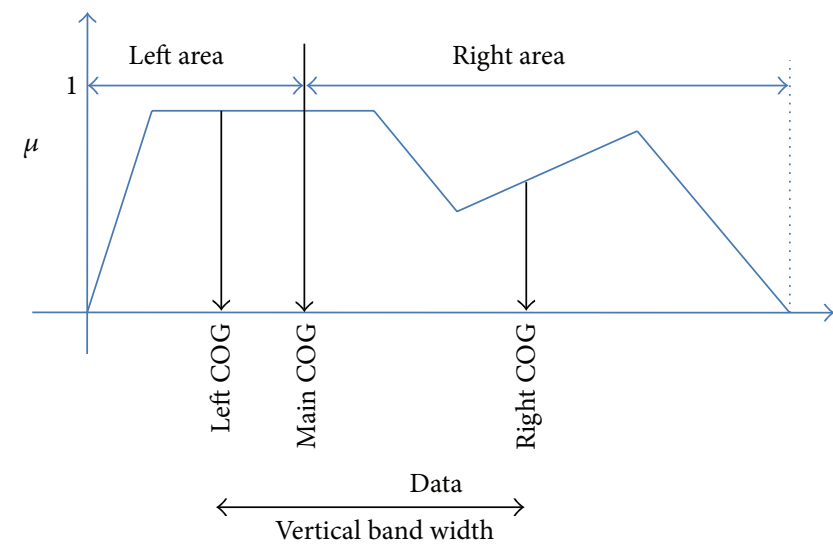

FIgURE 3: The concept of horizontal and vertical rule bases.

Figure 4 shows a single-input single-output process or control surface which is repositioned by vertical rules. Figure 5 shows a three-dimension prototype. In Figure 5 the colored surface is the main surface; this main surface can change to any other surfaces which depend on vertical rule base definitions. After applying vertical rule base, the obtained surfaces are complicated surfaces which are not possible to be implemented by few rules in traditional fuzzy systems. If the same work was done by traditional fuzzy systems apart from exploit large number of rules, describing the system behavior is more difficult than the proposed system. The privilege of this system to fuzzy type 2 systems is that modulating of type 1 control surface (here, horizontal control surface) is more manageable and not limited.

This method is applicable to all complicated systems which are controllable by fuzzy traditional fuzzy systems. On the other hand, due to the existence of concepts and reasons behind our proposed method, it provides a designer with more opportunities to manage parameters related to uncertainty in fuzzy systems conveniently in engineering and industrial affairs more convenient and more informed.

Here, a case study on controlling of steaming room temperature is studied theoretically and practically for showing the effectiveness of the proposed method.

\section{Steaming Room}

The autoclave system has one on-off input valve and one onoff output valve. The total block diagram of all under control system is shown in Figure 6. In this case, the output valve is set to low output flow just to have the same pressure as atmosphere pressure without losing significant energy. This paper considered the pressure as atmosphere pressure and follows the changes of temperature without changing the pressure.

The input of controller is temperature. This input is uncertain due to the type of sensors which are used in the autoclave and steaming room environments. Another uncertainty is regarding the corresponding word and crisp quantities [11]. Because of final cooling stage of the autoclave process, it is necessary to use water tap shown in Figure 6 in order to satisfy 


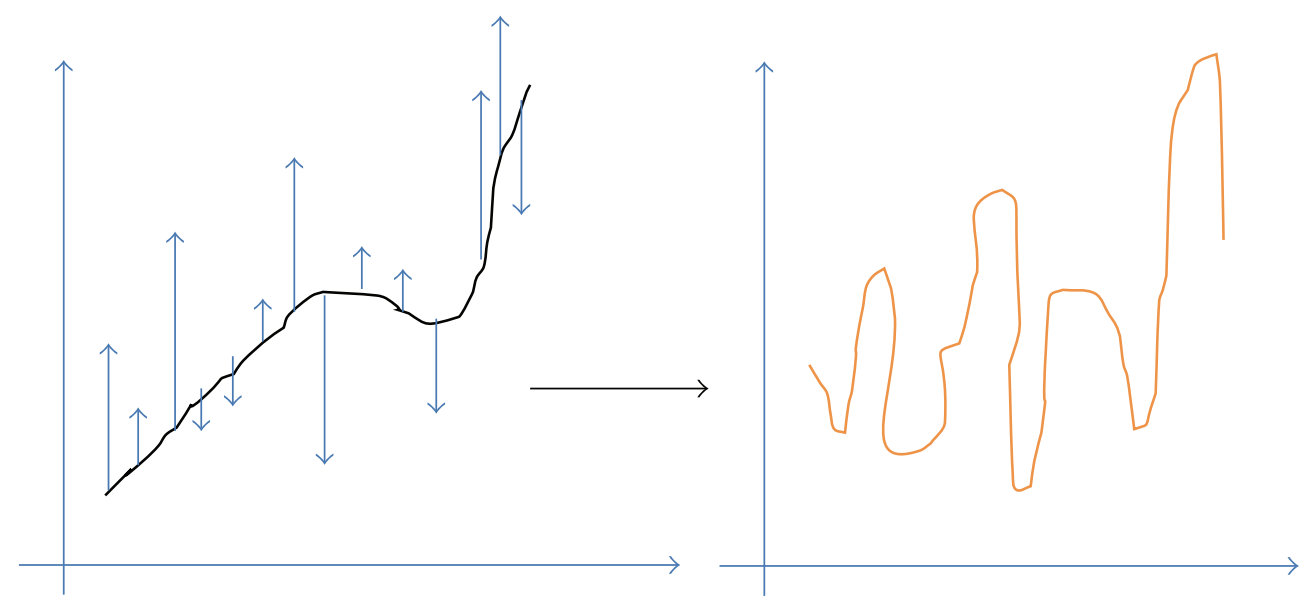

FIGURE 4: Modulating horizontal process surface by vertical rule base.

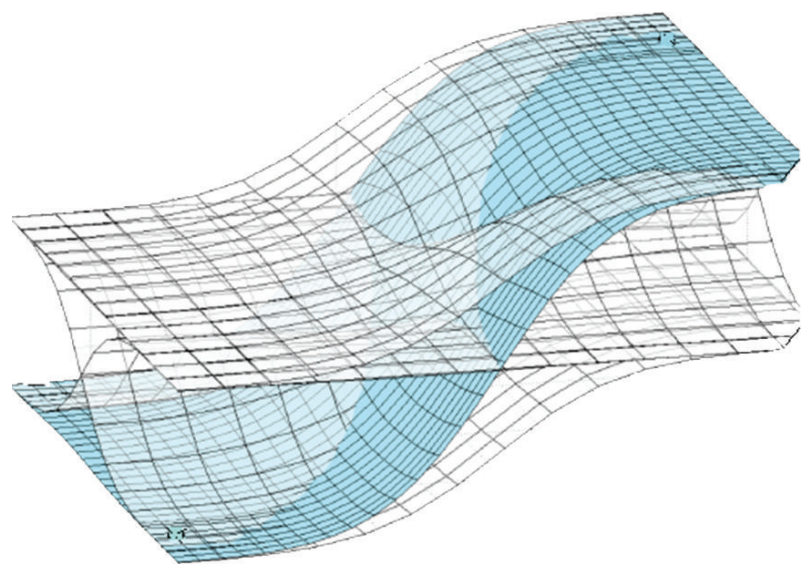

FIGURE 5: Blue (coloured) surface is related to horizontal rule base, others are possible final surfaces.

the optimum conditions. The vapor which the valve injects to system will cause change in temperature. The temperature sensor output also has nonlinear properties.

The autoclave system is controlled by two conventional methods: on-off and PID [16]. Simplicity and low cost are the advantages of on-off method but following the inconstant surfaces is difficult and also amortization of input valve is another drawback. In the other method, exact following of the level is an advantage of this method but it is complicated and expensive and valve amortization is high, too.

Another alternative is using fuzzy controller for managing the product process. The main point that we will involve in this case is that how we can define membership functions which are compatible with linguistic terms that conclude an output control sphere which is able to control the temperature of autoclave room so that we get better product quality and lesser wasting of input vapor.

The proposed controller wants to control the structure shown in Figure 6 for following two main purposes that are product high quality and energy efficiency which are both important in industries. In Figure 6 we can see one controller

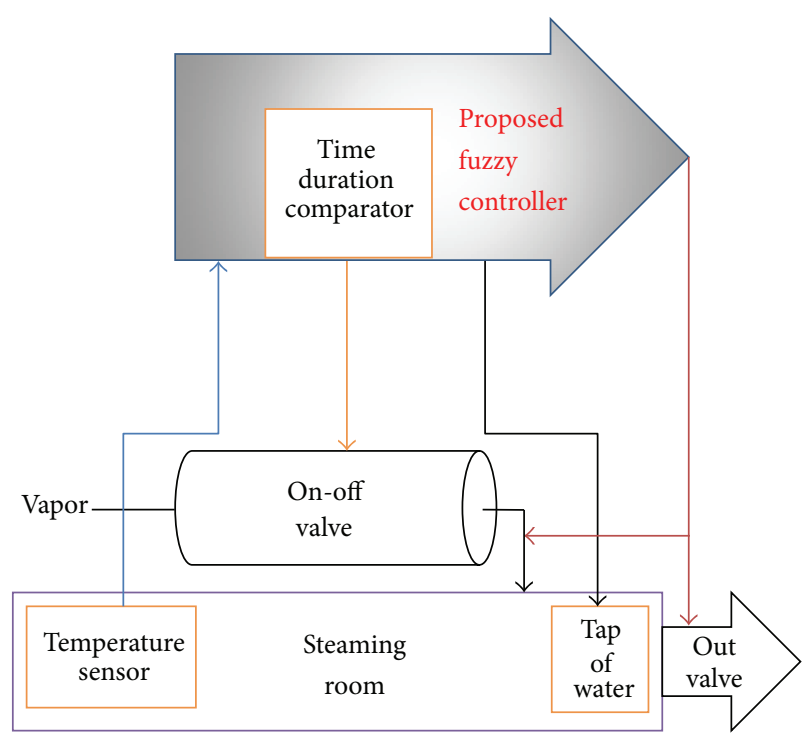

FIGURE 6: The practical block diagram of the under control system.

which involves with uncertain data of sensors and variation of parameters. The steaming room system is a system with high nonlinearity [17] and also involves with sensors and actuators which their parameters change and are not certain. This research involves with control of such a system with high nonlinearity and in difficulty to model it with classical methods.

The method of our controlling behavior here is under base of description and testing the system. Because of this at the beginning we do not have the curve which shows us the behavior of system which causes to reach the better quality.

As primitive information about the suitable curve that we have to achieve good product quality, there is just descriptive information about the curve which we can illustrate it as shown in Figure 7. It is necessary to know that this curve is not exact curve, and it just shows us that normally first we have increase of temperature and after that we have a flat zone with minimum temperature changes and finally the temperature 
must reduce in a specific time. In [1] the author says that in steaming room the temperature should start from 25 degree of centigrade and the temperature goes up linearly up to 95 degree of centigrade during 4 hours. After that it must stay in the temperatures around the last temperature for 3 hours. Finally, the cooling stage must start if needed using water tap.

\section{Proposed Fuzzy Structure and Its Implementation}

The main part of this controller is a fuzzy system which controls the autoclave behavior. This fuzzy system is constructed using min inference engine, triangular fuzzifier, and center of gravity (COG) defuzzifier. The controller is able to measure the temperature and set output flow of an on-off valve.

Three stages are needed to implement such a system described in Figures 2 and 5. The first stage is wordifier_better to say a higher type of fuzzifier which is able to model a word which describes the quantities. The second and third stages include decision maker and defuzzifying stage which provides a probabilistic space in output to limit the decision space toward wanted response.

Counting on the practical and field data, the only thing which we have at the first glance is information which is describable with the curve shown in Figure 7, but all the things are not limited to the above descriptions. For example, in the first stage of process which is related to the first four hours, at the beginning the increasing rate of temperature must be slower because the raw material must harden gradually for getting higher resistance and after two hours the increasing rate must intensify, because the raw material is harder than before. We do not want to bring all details here, because the aim of the paper is not to describe the details of chemical and physical properties of terrazzo tiles. For all this information we use field data of factory with consulting with related specialists. The aim of this paper is to show how we use these data and manage them to design our controller. If we consider the described suitable steaming room, two stages can be concluded usually in such processes if we emphasize on human knowledge descriptions: one stage which describes the total and main behavior of system which is shown in Figure 7. When we want to describe the system as an expert we describe the main behavior of system normally with the minimum rules. In our problem this part of system is the area between upper and lower bands shown in Figure 7. In the second stage after demonstrating the area using the preliminary description of system, we try to specify the optimum points in vertical axe between both upper and lower bands using secondary descriptions which related to details; one possible curve can be the preferred curve shown in Figure 7.

In order to implement this idea and in order to overcome uncertainty related to describing the words we proposed the system shown in Figure 8.

Figure 8 shows the block diagram of controller used to control an on-off valve with measuring temperature and control the duration time that valve is open or closed. If the sign of time is minus it means that for that time valve is closed

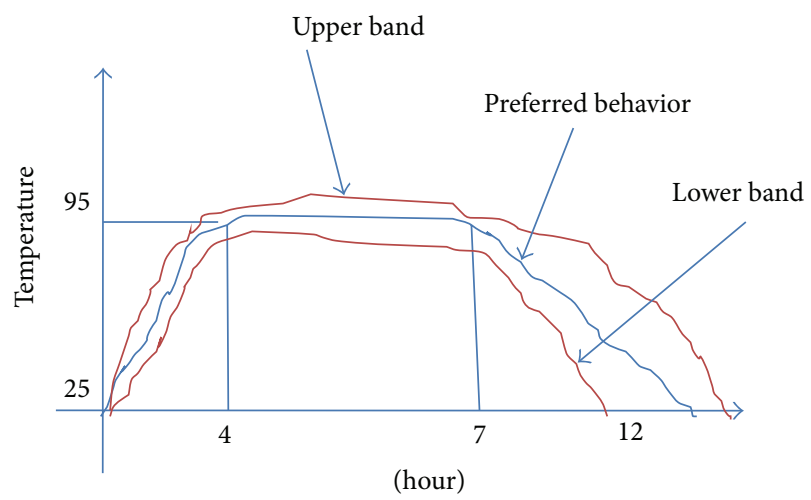

FIgURE 7: The approximate process curve.

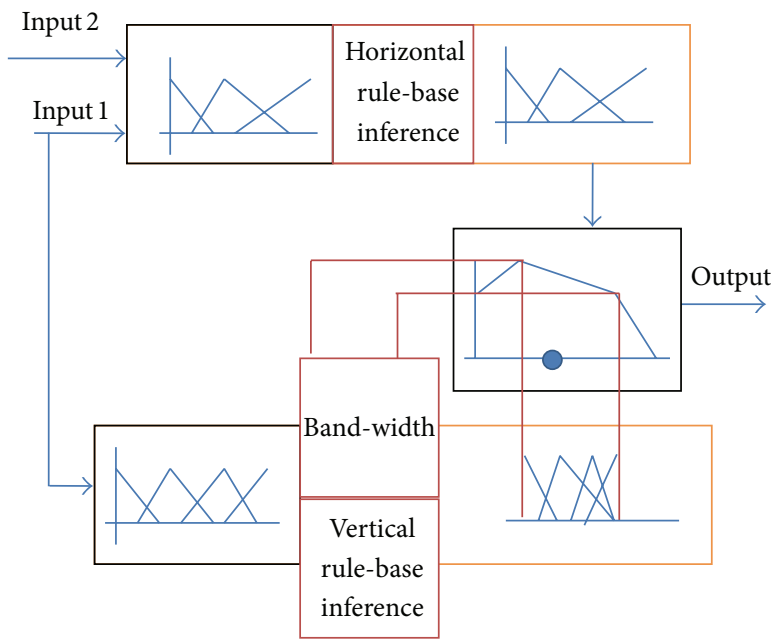

FIGURE 8: The total proposed block diagram.

otherwise is opened. In order to implement the main idea brought up in this paper and in order to overcome uncertainty related to describing the words we proposed the system shown in Figure 8 . The band width block in Figure 8 refers to band width which is shown in Figure 3.

To implement all steps a controller is needed which contains horizontal fuzzy block, vertical fuzzy block, and band width identifier block as shown in Figure 8. Figure 8 is the same as Figure 1 but with more details. A circuit should also be designed to change the information obtained from sensors into the accepted range by controller.

For describing the main rout we use just three rules as below (which we call them horizontal set of rules):

$\mathrm{H}$-Rule 1: If temperature error is very negative and temperature is low or medium, then the output duration time is high open.

$\mathrm{H}$-Rule 2: If temperature error is very negative and temperature is high, then the output duration time is low open.

$\mathrm{H}$-Rule 3: If temperature error is negative and temperature is low, then the output duration time is low open. 
H-Rule 4: If temperature error is negative and temperature is medium or high, then the output duration time is zero (closed or open).

$\mathrm{H}$-Rule 5: If temperature error is medium, then the output duration time is zero (closed or open).

$\mathrm{H}$-Rule 6: If temperature error is positive and temperature is medium or high, then the output duration time is zero (closed or open).

$\mathrm{H}$-Rule 7: If temperature error is positive and temperature is low, then the output duration time is low closed.

H-Rule 8: If temperature error is very positive and temperature is high, then the output duration time is low closed.

$\mathrm{H}$-Rule 9: If temperature error is very positive and temperature is low or medium, then the output duration time is high closed.

After that, we describe the deviation of main rout with another set of rules which we call them vertical rules. In this case first we consider the response of main rules (horizontal rules), after that, step by step by monitoring the system behavior or by using detailed knowledge which prepared earlier with present experts in site, and in each step we define necessary rule. In this case we used five rules for deviations from main rout. Of course we considered three ranges of deviation due to the property of the system under control.

V-Rule 1: If temperature is very low, then the output duration time is low and output band width is narrow.

V-Rule 2: If temperature is low, then the output duration time is medium and output band width is narrow.

V-Rule 3: If temperature is medium, then the output duration time is medium and output band width is narrow.

V-Rule 4: If temperature is high, then the output duration time is medium and output band width is medium.

V-Rule 5: If temperature is very high, then the output duration time is very high and output band width is wide.

The input range is divided to three intervals. These intervals must be clarified on the base of experienced and scientific knowledge of process under control. And after that we assign output partitions which our output here is the duration time that valve is open or closed.

Because of that the raw output of controller is the duration of time. The control signal first is applied to a timer system and by each new reading of temperature the output duration time is substituted with new output duration time. The controller system just in two statuses sends a command directly to on-off vale: (a) if the sign of recent duration time is opposite of the previous one and (b) while the duration time is expired.

DSP Implementation: A DSP (Model: TMS320VC5510 DSK Module) is used for implementing our controller. The total block diagram of VC5510 DSK is shown in Figure 10 [18]. Based Texas Instruments [18]. The 5510 DSK is a lowcost standalone development platform that enables users to valuate and develop applications for the TI C55XX DSP family. The DSK also serves as a hardware reference design for the TMS320VC5510 DSP. The structure is written on DSP (an Integrated Development Environment) developed by Texas Instruments.

The proposed structure is written on a DSP platform which is an Integrated Development Environment developed by Texas Instruments that provides an interface for its DSP. The written source code of the proposed structure is compiled using CCS so that it can be loaded and executed on the processor. The code runs wholly on the DSP once it is downloaded.

The inputs are supplied from temperature sensors in order to execute real-time simulation for the controlling of valve relay. The inputs to the ADC must be in the range of $0-3 \mathrm{~V}$. If the input values out of these ranges, it will spoil the converter on the processor.

Hence, the inputs are scaled down proportionally before it is supplied to the ADC as described in previous section. Alternatively, a current limiting/saturation threshold relay is required to be inserted before the current input post of the DSP to avoid burn-out. The first stage is devoted to show comparisons in the system's response in a feedback controller when we are using a type-1 FLC. The second stage is dedicated to test a traditional installed PID controller and for last session our proposed systems are applied to system and output data are achieved.

\section{Simulations and Results}

Figure 8 shows the feedback control system that was used for obtaining the simulation results of proposed method. The whole system was simulated in the MATLAB programming language, and the controller was designed to follow the input as closely as possible. The plant is a nonlinear system that is autoclave room temperature controlling.

For evaluating the transient closed-loop response of an autoclave control system we can use the same criteria that normally are used for adjusting constants in PID (Proportional Integral Derivative) controllers. These are the following.

(1) Integral of Square Error (ISE).

(2) Integral of the Absolute value of the Error (IAE).

(3) Integral of the Time multiplied by the Absolute value of the Error (ITAE).

The selection of the criteria depends on the type of response desired; the errors will contribute different for each criterion, so we have that large errors will increase the value of ISE more heavily than to IAE. ISE will favor responses with smaller overshoot for load changes, but ISE will give longer settling time. In ITAE, time appears as a factor, and therefore, ITAE will penalize heavily errors that occur late in time, but virtually ignore errors that occur early in time. Designing using ITAE will give us the shortest settling time, 


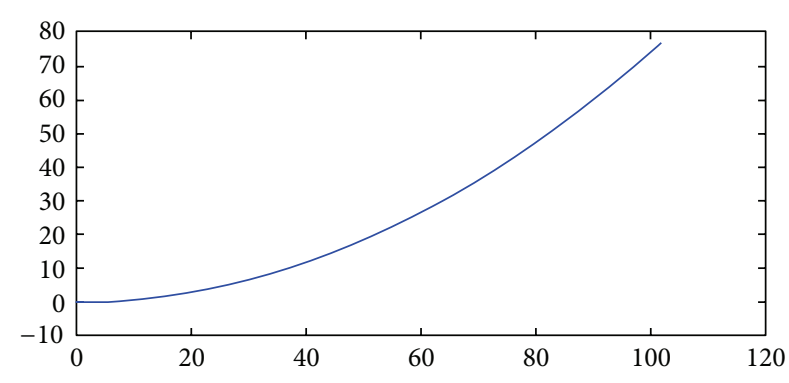

FIGURE 9: One possible simple curve which describes the nonlinearity behavior of autoclave system (descriptive approximation).

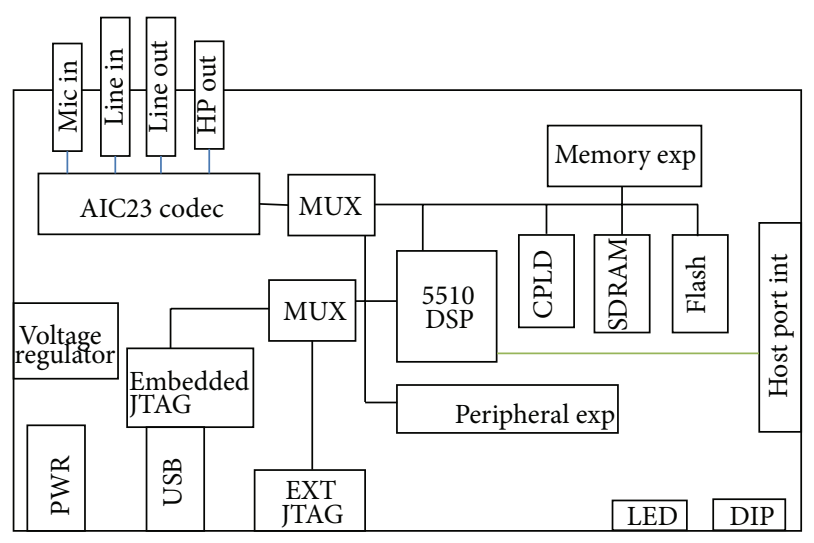

FIgURE 10: Block diagram VCC5510 DSK [18].

but it will produce the largest overshoot among the three criteria considered. Designing considering IAE will give us an intermediate result, in this case, the settling time will not be so large than using ISE or so small than using ITAE, and the same applies for the overshoot response.

For testing the rate of nonlinearity of our autoclave system, the specific and unchanged amount of vapor is injected to the system, by fixing the opening time of valve. This fix injection for different room internal temperatures, for example, $0,1,2,3, \ldots, 100$ as shown in Figure 9 is repeated. The horizontal axe of Figure 9 shows the room internal temperatures before vapor injection. The vertical axe shows the room temperature after equal vapor injection. Figure 9 shows a curve in which experiments started from special point. If start point changes, this curve will change because of that our under control system is nonlinear.

As described above the on-off valve is set on the minimum effective flow and the injected energy is controlled by the time which valve is open or closed. For achieving this purpose, proper membership functions for vertical rules of temperature error and open or closed time duration of valve versus seconds in horizontal rules are defined. Symmetric membership functions are applied for output of vertical rules.

Figure 11 shows the time duration to temperature error curve in the absence of temperature parameter (Input 2 in Figure 8). In Figure 11 it is clear that vertical rules base provided a more complicated curve which is using proper

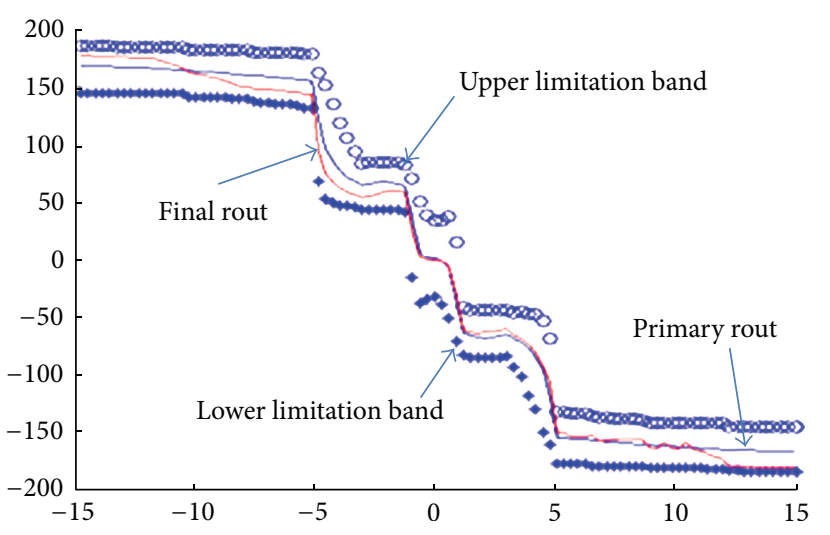

FIgURE 11: Primary, final routs and both bands in the absence of temperature parameter.

ANFIS, and this curve was extracted with 25 and 10 membership functions for input and output, respectively. The same curve is obtained here with very lesser numbers and with a descriptive method.

Considering that the temperature parameter and its relation to the injection time duration is a vertical relation, Figure 12(a) shows control surface of process. Figure 12(b) shows the final control surface which is complicated than Figure 12(a) shapes which show primary control surface.

In Figure 13(a) we see the difference values between final and primary control surfaces. Figure 13(b) shows the points which their final surface is under primary surface.

Applying final control surface to autoclave model approximation showed better results than primary control surface in point of view of number of switching of valve and expected output rout. Also, the same comparison was done with obtained practical data obtained from installed PID controller. Considering that the PID controllers lack facility to describe unknown system, the results show that our controller provides better protection of the material inside the autoclave room from unwanted temperature shocks.

Figure 14 shows two different step responses, first one for type 1 fuzzy system which is constructed by using horizontal fuzzy system only and second one by using total fuzzy system proposed in Figure 8. As we see in Figure 14 the vertical rule base improves the output quality.

We have presented the design of the controllers design for nonlinear control system using horizontal and vertical fuzzy rule bases, as well as a comparative study in nonlinear system identification addressing the problem of autoclave controlling using ANFIS.

In the experiments a quantification of errors was achieved and documented in Table 1 for different criteria such as ISE, IAE, and ITAE, it was shown that the lower overshoot errors and the best settling times were obtained using horizontal and vertical rule base FLC, and the performance of this kind of controller is better for high noise level, concluding that it is a better option to use HV Fuzzy System model than traditional fuzzy systems.

For the second study, using ANFIS modeling, we found that after choosing the best membership functions for horizontal and vertical rule bases, the error was lower than ANFIS 


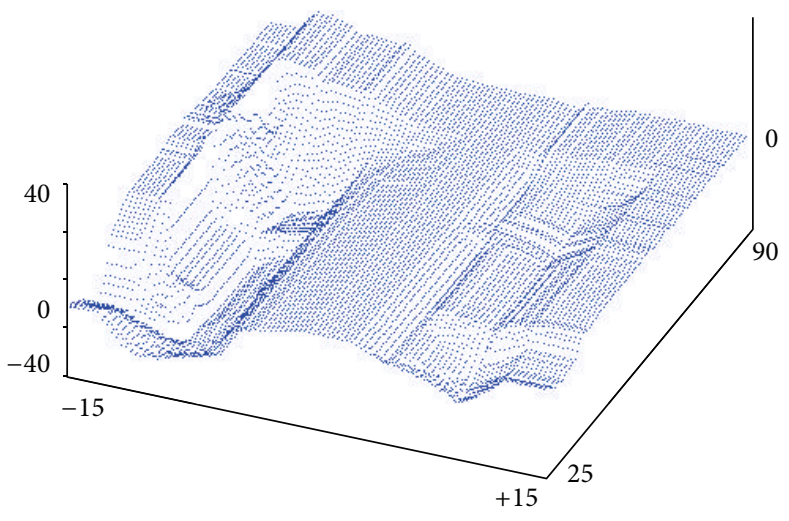

(a)

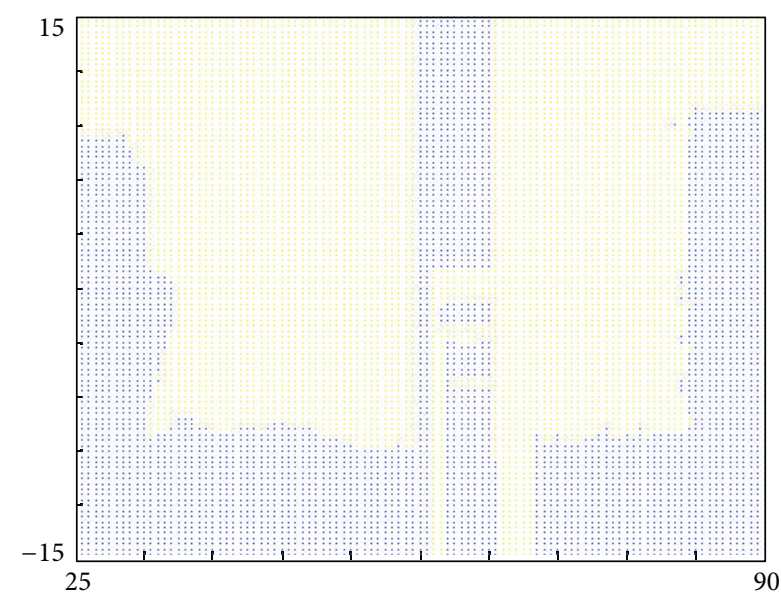

(b)

FIGURE 12: Control surface of process: (a) the primary control surface which is complicated than (b) the final control surface.

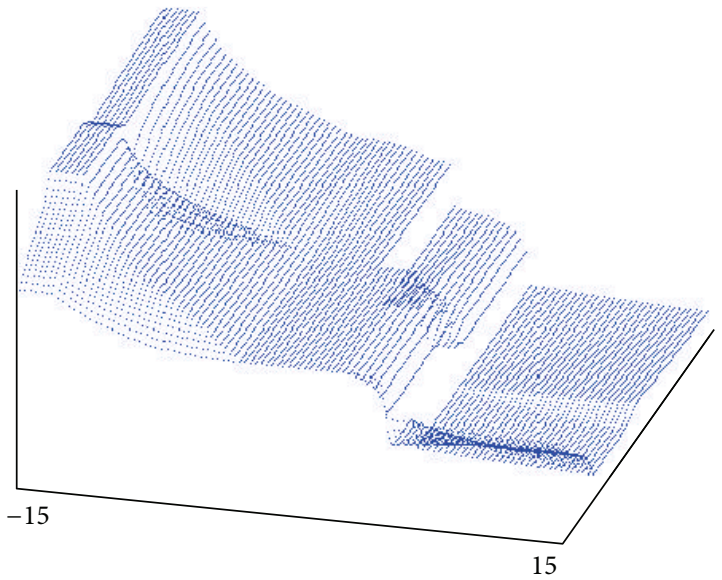

(a)

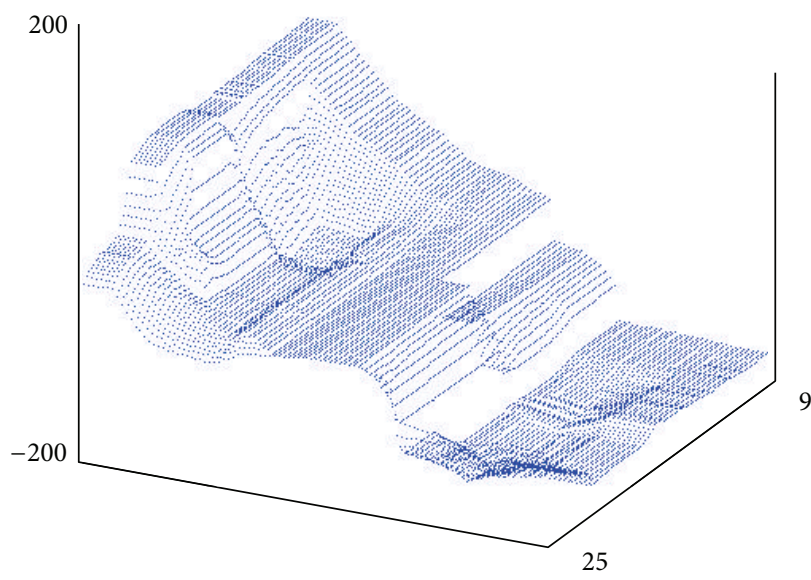

(b)

FIGURE 13: (a) Blue dot area = final surface, and yellow area = primary surface (b) (final surface)-(primary surface).

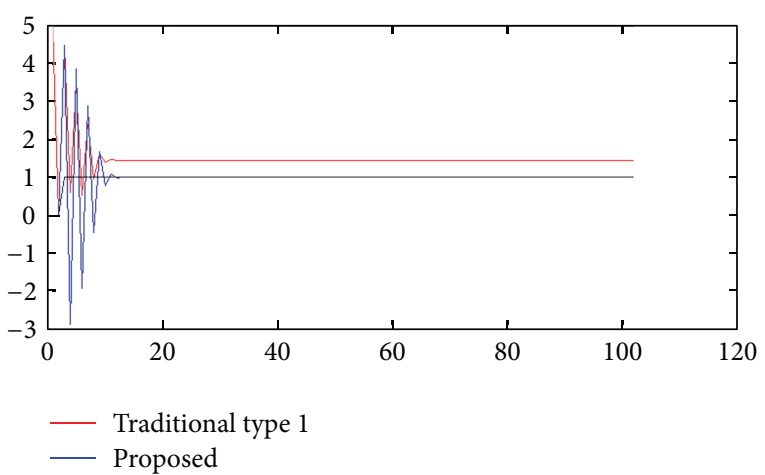

FIGURE 14: Step responses of horizontal type 1 fuzzy system and our total proposed system.

modeling. The difference is remarkable with the practical data, this is with data which was not used to train the system, somehow uncertain data. Here is where we can see, based on the experimental results, that the behavior of horizontal and vertical rule bases was better than ANFIS modeling.

For both studies, we can say that the best results were obtained using horizontal and vertical fuzzy systems. In our opinion this is because horizontal and vertical fuzzy sets that are used in our proposed fuzzy systems can handle uncertainties in a better way because they provide us with more parameters and more design degrees of freedom.

From the results obtained, proposed DSP system shows its competency in achieving minimum times to turn on the valve. Thus, the numerical data is able to prevent mal-trip and at the same time maintain the coordination requirement with reliability. As a result, the proposed method is very suitable for overcome uncertainties which require high reliability, sensitivity, selectivity, and speed.

As shown in Table 1 our proposed structure (Figure 8) outperformed traditional fuzzy systems and PID controller which practically installed in artificial stone factory on 
TABLE 1: Comparison of system performances.

\begin{tabular}{|c|c|c|c|c|c|}
\hline Performance criteria & $\begin{array}{l}\text { Installed traditional } \\
\text { controller in the site }\end{array}$ & $\begin{array}{c}\text { Type } 1 \text { main } \\
\text { approximator } \\
\text { Matlab simulation }\end{array}$ & $\begin{array}{c}\text { Type } 1 \text { main } \\
\text { approximator DSP } \\
\text { module in factory } \\
\text { (practical) }\end{array}$ & $\begin{array}{l}\text { Horizontal and } \\
\text { vertical system } \\
\text { proposed structure } \\
\text { Matlab simulation }\end{array}$ & $\begin{array}{l}\text { Horizontal and } \\
\text { vertical system } \\
\text { proposed structure } \\
\text { factory (practical) }\end{array}$ \\
\hline ISE/IAE/ITAE & - & $7.65 / 18 / 383$ & - & $5.1 / 17.3 / 320$ & - \\
\hline $\begin{array}{l}\text { Total duration of closed } \\
\text { valve }\end{array}$ & 90 & 95 & 85 & 89 & 77 \\
\hline $\begin{array}{l}\text { Total times of valve } \\
\text { switching }\end{array}$ & 313 & 297 & 302 & 193 & 220 \\
\hline $\begin{array}{l}\text { Closed average time } \\
\text { duration }\end{array}$ & 0.57 & 0.63 & 0.56 & 0.92 & 0.70 \\
\hline
\end{tabular}

the autoclave room. As it is clear, looking at Table 1, we see the amortization of valve significantly decreases using our proposed method.

There is mathematical calculations on stability concerns in [19] for the problem of exponential stability analysis for distributed time varying delays. In our proposed work, it has been practically checked stability of system for several specific statuses. The proposed system easily is applicable to systems with uncertain and unforeseeable data such as [20-23] and it also has great ability to be used as an alternative in such conditions investigated in [24-26]. The proposed method easily is implementable by hardware structures in [15].

\section{Conclusion}

This paper describes a new method of uncertainty description in fuzzy controllers by introducing the concept of horizontal and vertical rule bases in fuzzy controllers and their roll in easing of extracting the optimum control surface by using too lesser rules than traditional fuzzy systems. From the results obtained, proposed DSP system shows its competency in achieving minimum time to control valve and is able to detect transient input. As a result, the proposed method is very suitable for overcoming uncertainty which its handling requires high reliability, sensitivity, selectivity, and speed. The power of proposed method to simplify description of complicated systems is shown by applying this method to control a steaming room system to reduce input valve amortization and setting the temperature of the autoclave room at defined points which guide us to reach better product quality of artificial stones and prevent misusing energy. Simulations confirm the lesser number of valve switching and better energy saving by applying proposed controller to the under control process compared to traditional counterparts and smoother rout compare to PID controllers.

We showed the effective ability of this method in order to describe the system on the base of observations of system behavior. On the other hand, due to the existence of concepts and reasons behind our proposed method, it provides a designer with more opportunities to manage parameters related to uncertainty in fuzzy systems conveniently in engineering and industrial affairs more convenient and more informed.
Finally, the paper confirms achieving better results of proposed method by doing simulation on steaming room model in artificial stone maker factory, and providing more compatible control surface to create better tool for system behavior description.

\section{Acknowledgments}

The authors would like to acknowledge the support of Universiti Sains Malaysia and the first author would like to acknowledge the support of USM fellowship.

\section{References}

[1] S. Aminifar and A. b. Marzuki, "A Novel fuzzy controller applicable to steaming room," in Proceedings of the IEEE International Conference on Control System (SMC '12), Computing and Engineering, Batufiringi, Pulau Pinang, Malaysia, November 2012.

[2] R. Yang, P. Shi, and G. P. Liu, "Filtering for discrete-time networked nonlinear systems with mixed random delays and packet dropouts," Institute of Electrical and Electronics Engineers, vol. 56, no. 11, pp. 2655-2660, 2011.

[3] X. Su, P. Shi, L. Wu, and Y. D. Song, "A novel control design on discrete-time takagi-sugeno fuzzy systems with time-varying delays," IEEE Transactions on Fuzzy Systems, no. 99, Article ID 2226941, 2012.

[4] X. Su, P. Shi, L. Wu, and Y. D. Song, "A novel approach to filter design for T-S fuzzy discrete-time systems with time-varying delay," IEEE Transactions on Fuzzy Systems, vol. 20, no. 6, pp. 1114-1129, 2012.

[5] X. Su, P. Shi, L. Wu, and S. K. Nguang, "Induced $\ell_{2}$ filtering of fuzzy stochastic systems with time-varying delays," IEEE Transactions on Systems, Man, Cybernetics B, no. 99, Article ID 2227721, pp. 1-14, 2012.

[6] X. Su, L. Wu, P. Shi, and Y. D. Song, " $H_{\infty}$ model reduction of TS fuzzy stochastic systems," IEEE Transactions on Systems, Man, and Cybernetics B, vol. 42, no. 6, pp. 1574-1585, 2012.

[7] H. Peng and M. Zhou, "Quantity discount supply chain models with fashion products and uncertain yields," Mathematical Problems in Engineering, vol. 2013, Article ID 895784, 11 pages, 2013.

[8] X. W. Tang, Y. D. Zhou, and Y. L. Liu, "Factors influencing quasistatic modeling of deformation and failure in rock-like solids by the smoothed particle hydrodynamics method," Mathematical Problems in Engineering, vol. 2013, Article ID 852875, 13 pages, 2013. 
[9] R. Yuan-Yuan, Z. Hong-Wei, L. Xian-Sheng, and Z. Xue-Lian, "Study on vehicle track model in road curved section based on vehicle dynamic characteristics," Mathematical Problems in Engineering, vol. 2012, Article ID 818136, 17 pages, 2012.

[10] M. H. Hsu, "Dynamic analysis of cracked cantilever, electrostatic microactuators using radial basis functions," Mathematical Problems in Engineering, vol. 2012, Article ID 865461, 11 pages, 2012.

[11] J. M. Mendel and R. I. John, "Type-2 fuzzy sets made simple," IEEE Transactions on Fuzzy Systems, vol. 10, no. 2, pp. 117-127, 2002.

[12] D. Wu and J. M. Mendel, "Uncertainty measures for interval type-2 fuzzy sets," Information Sciences, vol. 177, no. 23, pp. 5378-5393, 2007.

[13] D. Wu and J. M. Mendel, "A comparative study of ranking methods, similarity measures and uncertainty measures for interval type-2 fuzzy sets," Information Sciences, vol. 179, no. 8, pp. 1169$1192,2009$.

[14] L. Gao and Y. Dai, "Research on system modeling and control of turbine-driven centrifugal ompressor," in Proceedings of the 6th IEEE Conference on Industrial Electronics and Applications (ICIEA '11), pp. 2090-2095, June 2011.

[15] S. Aminifar, A. Khoei, K. H. Hadid, and G. H. Yosefi, "A digital CMOS fuzzy logic controller chip using new fuzzifier and max circuit," International Journal of Electronics and Communications, vol. 60, no. 8, pp. 557-566, 2006.

[16] O. Ekren, S. Sahin, and Y. Isler, "Operation of compressor and electronic expansion valve via different controllers," in Fuzzy Logic-Controls, Concepts, Theories and Applications, InTech.

[17] G. Haigang, L. Hongxing, Z. Weijing, and S. Zhankui, "Direct adaptive fuzzy sliding mode control with variable universe fuzzy switching term for a class of MIMO nonlinear systems," Mathematical Problems in Engineering, vol. 2012, Article ID 543039, 21 pages, 2012.

[18] Texas Instruments TMS320VC5510 DSP CPU Reference Guide, 2002, http://c5000.spectrumdigital.com/dsk5510/docs/dsk5510_ techref.pdf.

[19] R. Yang, Z. Zhang, and P. Shi, "Exponential stability on stochastic neural networks with discrete interval and distributed delays," IEEE Transactions on Neural Networks, vol. 21, no. 1, pp. 169-175, 2010.

[20] H. Ying-Chin, H. Wen-Chieh, and C. Cheng-Chang, "Multicategory and multi-standard project selection with fuzzy valuebased time limit," International Journal of Innovative Computing, Information and Control, vol. 9, no. 3, pp. 971-989, 2013.

[21] T. Niknam, H. D. Mojarrad, and M. Nayeripour, "A new hybrid fuzzy adaptive particle swarm optimization for nonconvex economic dispatch," International Journal of Innovative Computing, Information and Control, vol. 7, no. 1, pp. 189-202, 2011.

[22] C. T. Su, F. F. Wang, and T. Yang, "Evolving optimal fuzzy-connective-based hierarchical aggregation networks using genetic algorithms," International Journal of Innovative Computing, Information and Control, vol. 8, no. 1, pp. 617-631, 2012.

[23] T. Chen, "A hybrid fuzzy and neural approach with virtual experts and partial consensus for dram price forecasting," International Journal of Innovative Computing, Information and Control, vol. 8, no. 1, pp. 583-597, 2012.

[24] L. Chih-Min, H. Chun-Fei, and Y. Rong-Guan, "Adaptive fuzzy sliding-mode control system design for brushless DC motors," International Journal of Innovative Computing, Information and Control, vol. 9, no. 3, pp. 1259-1270, 2013.
[25] M. Nafar, G. B. Gharehpetian, and T. Niknam, "Using modified fuzzy particle swarm optimization algorithm for parameter estimation of surge arresters models," International Journal of Innovative Computing Information and Control, vol. 8, no. 1, pp. 567-581, 2012.

[26] T. T. Gang, J. Yang, Q. Gao, Y. Zhao, and J. Qiu, "A fuzzy approach to robust control of stochastic nonaffine nonlinear systems," Mathematical Problems in Engineering, vol. 2012, Article ID 439805, 17 pages, 2012. 


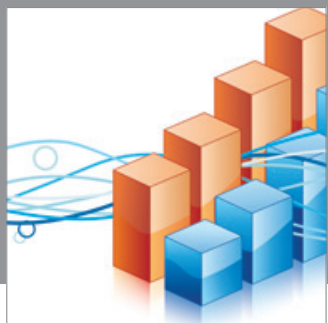

Advances in

Operations Research

mansans

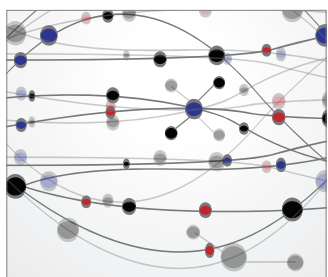

The Scientific World Journal
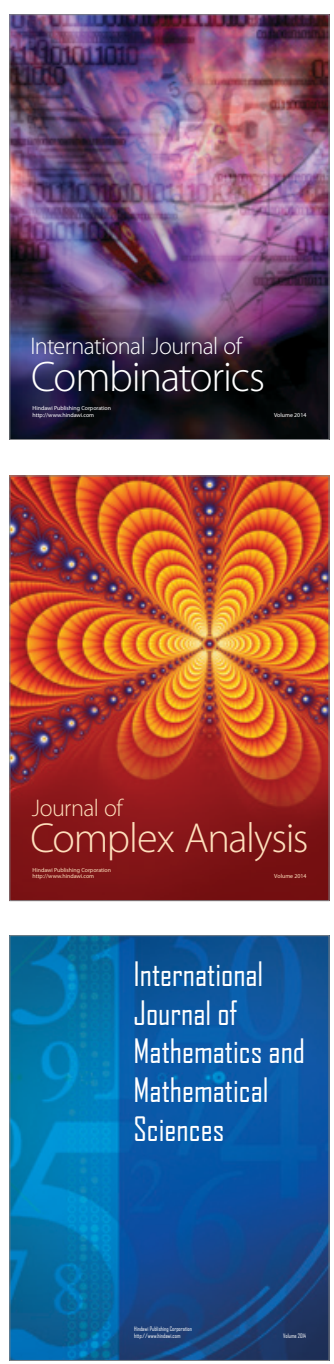
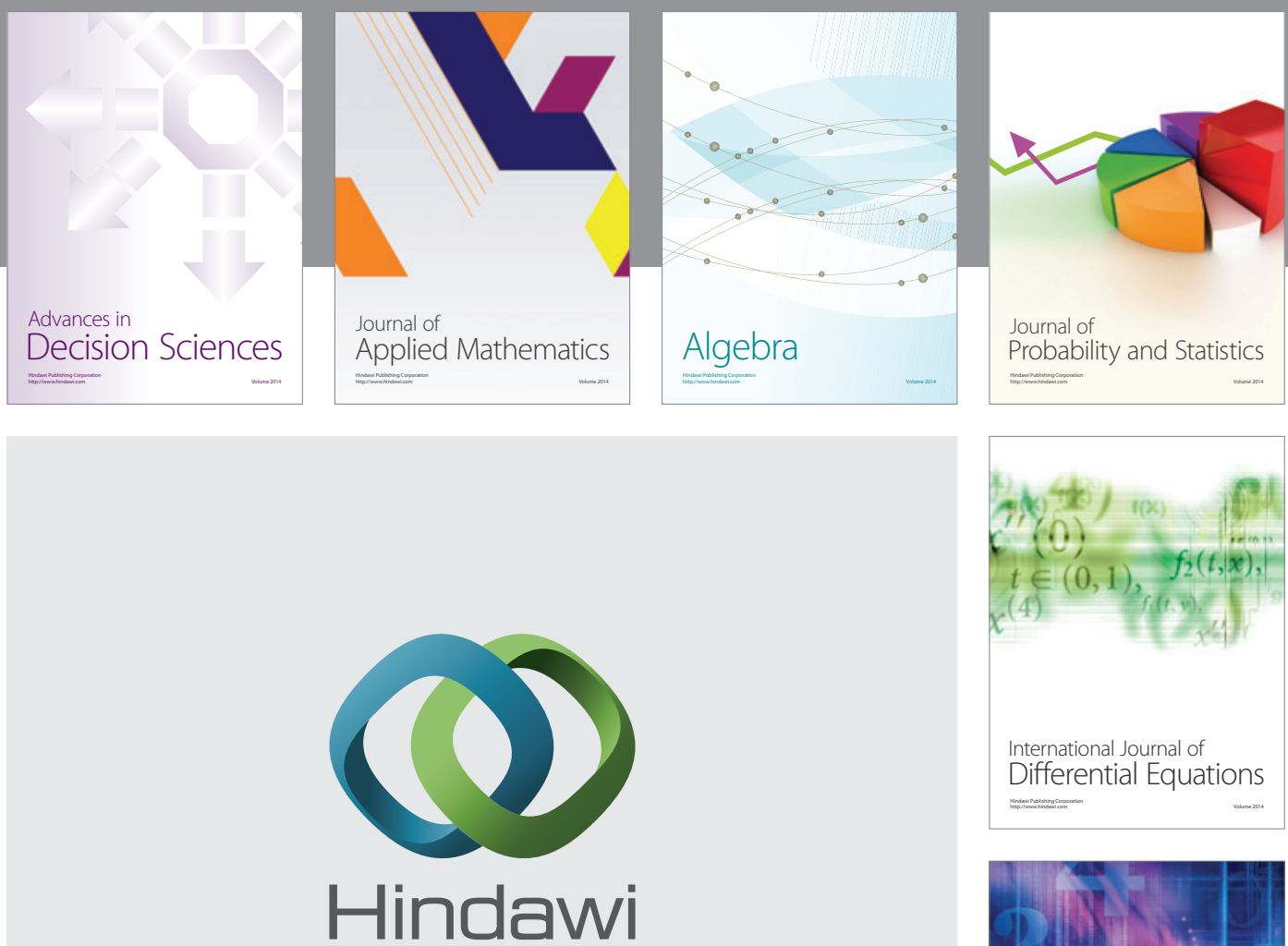

Submit your manuscripts at http://www.hindawi.com
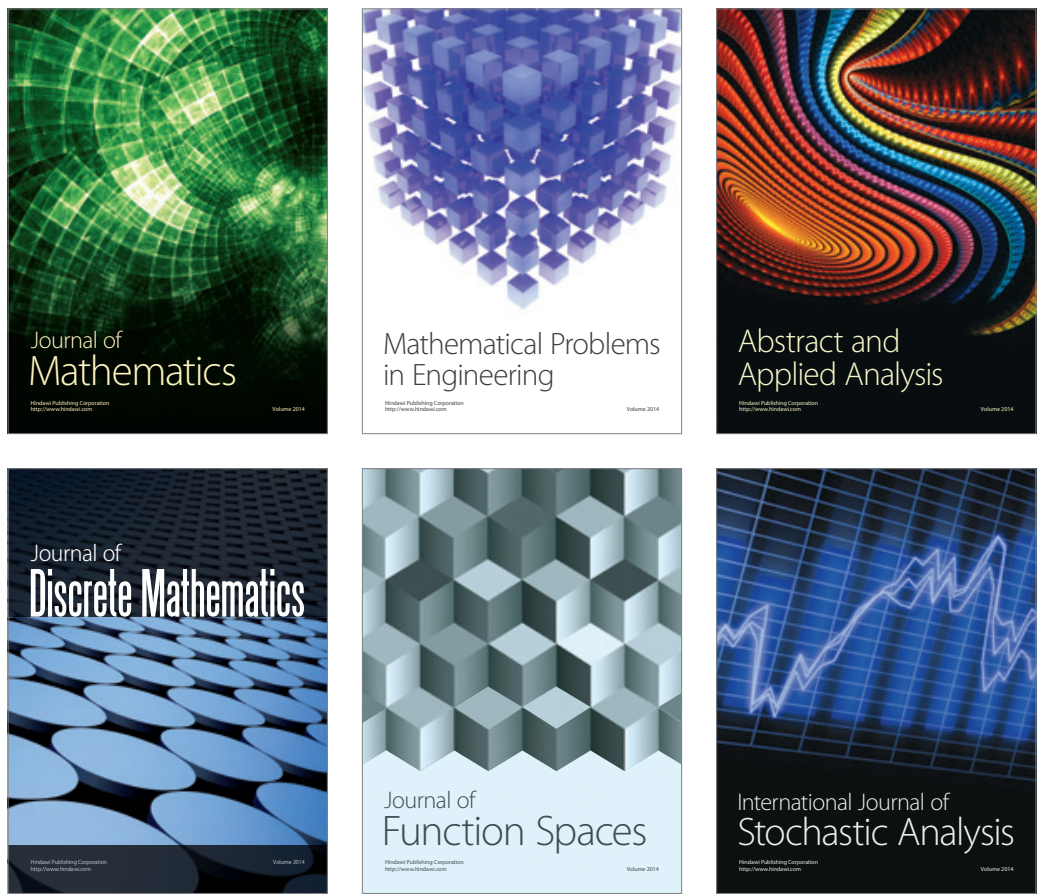

Journal of

Function Spaces

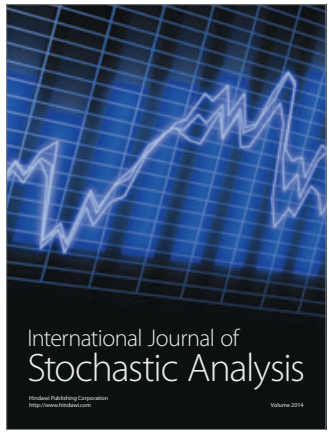

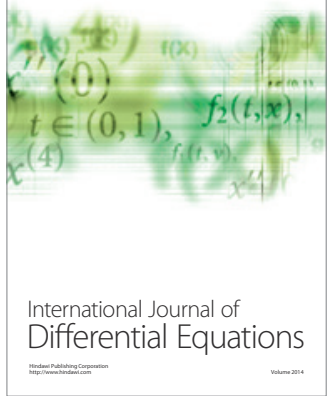
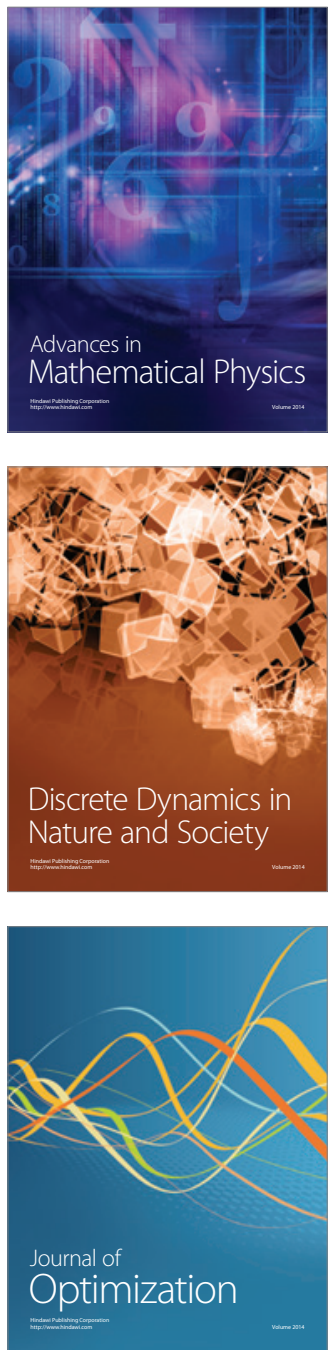